\title{
MHD Mixed Convection Flow from a Vertical Plate Embedded in a Saturated Porous Medium with Melting and Heat Source or Sink
}

\author{
*M.V.D.N.S.Madhavi ${ }^{1}$, J.Siva Ram Prasad ${ }^{2}$ K.Hemalatha ${ }^{2}$, , \\ ${ }^{I}$ Scholar, Krishna University, Dept. of Mathematics, V.R.Siddhartha Engineering College, Vijayawada, India, \\ ${ }^{2}$ Dept. of Mathematics, V.R.Siddhartha Engineering College, Vijayawada, India
}

\begin{abstract}
We analysed in this paper the problem of MHD mixed convection flow from a vertical plate embedded in a saturated porous medium in the presence of melting, thermal dispersion, radiation and heat absorption or generation effects for aiding and opposing external flows. Similarity solution for the governing equations is obtained for the flow equations in steady state. The equations are numerically solved by Runge-Kutta fourth order method coupled with shooting technique. The effect of melting and heat absorption or generation under different parametric conditions on velocity, temperature and heat transfer was analyzed for both aiding and opposing flows.
\end{abstract}

Keywords: Heat absorption or generation, Melting, MHD, Porous medium, Radiation, Thermal dispersion.

\section{INTRODUCTION}

Over the last few years the study of heat transfer with melting effect in porous media has been increased due to wide variety of applications in industry such as magmasolidification, melting of the permafrost, preparation of semiconductor material. Roberts [1] was the first to study the shielding effect to describe the melting phenomena of ice placed in ahot stream of air in the steady. Transport in porous media has received continuing interest in the past five decades. This interest stems from their importance in many industrial and clinical applications. Because of these applications, several investigators have turned their attention to the study of fundamental and applied problems related to heat transfer in porous media. Also the problem of combined free and forced convection (mixed convection) in a porous medium has many important applications in geothermal reservoirs where pressure gradients may be generated either by artificial with drawl or injection of fluids or by natural recharge or discharge of meteoric water. $\mathrm{M}$ Kazmierczaketal [2] and $\mathrm{P}$ Cheng[3]studied melting from a flat plate embedded in a porous medium in the presence of natural convection, combined free and forced boundary layer flows about inclined surfaces. Merkin [4] has considered mixed convection boundary-layer flow in porous media adjacent to a vertical uniform heat flux surface.

Due to the important and interesting applications in geothermal energy extraction, nuclear waste disposal industry, underground heat exchangers for energy storage and recovery, temperature controlled reactors, packed beds and the utilization of porous layers for transpiration cooling by water for fire fighting, in the storage of food grains, etc., the study of convective heat transfer in a non-Darcy porous medium has been gaining the attention of several researchers. Chamkha [5] presented a numerical study for nonDarcy hydro magnetic free convection flow of an electrically-conducting and heat-generating fluid over a vertical cone and a wedge adjacent to a porous medium. Murthy and Singh [6] analyzed thermal dispersion effects on non-Darcy convection over a cone. An analysis is performed by Alin v. Roscaetal [7]to study the heat and mass transfer characteristics of mixed convection flow along a vertical plate embedded in a fluid saturated porous medium under the combined buoyancy effects of thermal and mass diffusion. They showed that dual solutions exist for a certain range of parameters in the problem. Very interesting analytical solutions have also been included in the paper.A note on the effect of surface melting on the steady mixed convection boundary layer flow over avertical flat surface embedded in afluid saturated porous medium is studied further by J.H.Merkin etal[8] which is previously studied by Ahmad and Pop[9]. The main conclusion is that solutions are possible only if $M<1$ with the limit as $m \rightarrow 1$ being discussed. The critical values, identified in Ahmad and Pop[9] are examined in more detail and the free convection limit derived.

When dealing problems in porous media, the effects of melting, radiation and heat absorption or generation become important. The problem of unsteady mixed convection boundary layer flow near the stagnation point on a heated vertical plate embedded in a fluid saturated porous medium with thermal radiation and variable viscosity was investigated by Hassanien and Al-arabi [10]. Murthy et al. [11] considered mixed convection 
flow of an absorbing fluid up a uniform non-Darcy porous medium supported by a semi-infinite ideally transparent vertical flat plate due to solar radiation. Chamkha et al. [12] presented a numerical study of coupled heat and mass transfer by boundary-layer free convection over a vertical flat plate embedded in a fluid-saturated porous medium in the presence of thermophoretic particle deposition and heat generation or absorption effects. The effects of Non-Darcy mixed convection with thermal dispersion-radiation in a saturated porous medium was studied by Prasad and Hemalatha [13]. They observed that temperature decreases with increasing melting parameter.Chamkha [14] discussed heat and mass transfer for a nonNewtonian fluid flow along a surface embedded in a porous medium with uniform wall heat and mass fluxes and heat generation or absorption. AlsoChamkha et al [15] analyzed melting and radiation effects on mixed convective flow from a vertical surface embedded in a non-Newtonian fluid saturated non-Darcy porous medium for aiding and opposing external flows. They obtained representative flow and heat transfer results for various combinations of physical parameters. Motivated by the works mentioned above, the present paper aims at analysing the effect of melting, thermal dispersion-radiation and heat absorption and generation on mixed convection from a vertical plate embedded ina saturated porous medium for aiding and opposing external flows

\section{MATHEMATICAL FORMULATION}

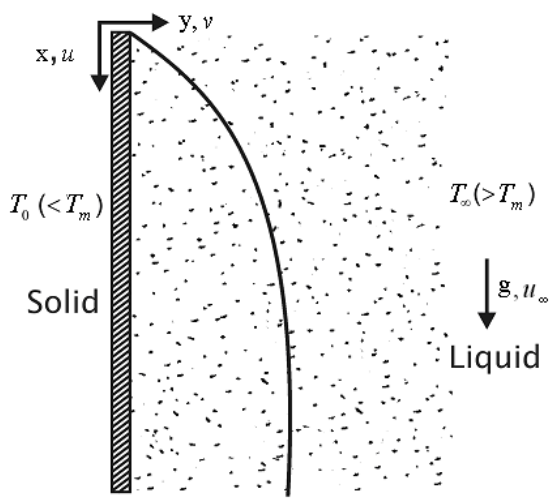

Fig. 1 Schematic diagram of the problem.

We considered a steady mixed convection boundary layer flow past a vertical melting surface embedded in a fluid saturated porous medium. The flow model and geometry are shown in the figure1. Further we consider a Cartesian coordinate system $(\mathrm{x}, \mathrm{y})$, where $\mathrm{x}$ and $\mathrm{y}$ are coordinates measured along the plate and normal to it, respectively. In this coordinate system it appears as if the frozen porous medium moves towards the stationary melting/solid interface with constant velocity equal to the melting velocity (The melting front is modelled as a vertical plate. This plate constitutes the inter phase between the liquid phase and the solid phase during melting inside the porous matrix. The temperature of the solid region is considered less than the melting point, i.e., $T_{0}<T_{m}$. On the right hand side of melting front, the liquid is super- heated, i.e., $\mathrm{T}_{\mathrm{m}}<\mathrm{T}_{\infty}$. A vertical boundary layer flow, on the liquid side smoothes the transition from $\mathrm{T}_{\mathrm{m}}$ to $\mathrm{T}_{\infty}$. The assisting external flow velocity is taken as $\mathrm{u}_{\infty}$.

When taking into consideration the effect of thermal dispersion and thermal radiation, the governing equations for steady non-Darcy flow in aporous medium can be written as follows.
The continuity equation is $\frac{\partial u}{\partial x}+\frac{\partial v}{\partial y}=0$ (1)

The momentum Equation is

$\frac{\mu}{\mathrm{K}} \frac{\partial u}{\partial y}+\frac{\mu}{\mathrm{K}} \frac{\mathrm{C} v \mathrm{R}}{\mathrm{y}} 2 \mathrm{u} \frac{\partial \mathrm{u}}{\partial y}+\sigma \mathrm{B}_{0}^{2} \frac{\partial u}{\partial y}=-\rho_{m} g \beta \frac{\partial \mathrm{T}}{\partial y}$

$\begin{array}{lc}\text { The } & \begin{array}{c}\text { Energy } \\ \mathrm{u} \frac{\partial \mathrm{T}}{\partial \mathrm{x}}+\mathrm{v} \frac{\partial \mathrm{T}}{\partial \mathrm{y}}=\frac{\partial}{\partial y}\left(\alpha \frac{\partial \mathrm{T}}{\partial \mathrm{y}}\right)-\frac{1}{\mathrm{p} \mathrm{C}_{\mathrm{p}}} \frac{\partial \mathrm{q}}{\partial \mathrm{y}}+\frac{\alpha \mathrm{Q}_{0}}{\mathrm{k}}\left(\mathrm{T}-\mathrm{T}_{\mathrm{m}}\right)\end{array}\end{array}$

Here, $\mathrm{u}$ and $\mathrm{v}$ are the velocities along $\mathrm{x}$ and $\mathrm{y}$ directions respectively, $\mathrm{T}$ is temperature in the thermal boundary layer, $\mathrm{K}$ is Permeability, $\mathrm{k}$ is thermal conductivity, $\mathrm{B}_{0}$ Magnetic field strength, $\mathrm{C}$ is Forchheimer empirical constant, $\beta$ is coefficient of thermal expansion, $v$ is kinematics viscosity, $\rho$ is density, $\mu$ Viscosity, oelectrical conductivity, $C_{p}$ is specific heat at constant pressure, $\mathrm{g}$ is acceleration due to gravity, and thermal diffusivity $\alpha=\alpha_{\mathrm{m}}+\alpha_{\mathrm{d}}$, where $\alpha_{m}$ is the molecular diffusivity and $\alpha_{d}$ is the dispersion thermal diffusivity due to mechanical dispersion, $\mathrm{Q}_{0}$ is the volumetric heat generation or absorption parameter. As in the linear model proposed by Plumb [16] the dispersion thermal diffusivity $\alpha_{d}$ is proportional to the velocity component i.e. $\alpha_{d}=\gamma u d$, where $\gamma$ is the dispersion 
coefficient and $d$ is the mean particle diameter. The radiative heat flux term $\mathrm{q}$ is written using the Rosseland approximation (Sparrow and Cess [17], Raptis [18] as

$$
\mathrm{q}=-\frac{4 \sigma_{\mathrm{R}}}{\mathrm{ag}} \frac{\partial \mathrm{T}^{4}}{\partial \mathrm{y}}
$$

Where $\sigma_{\mathrm{R}}$ is the Stefan-Boltzmann constant and ' $\mathrm{a}$ ' is the mean absorption coefficient.

The physical boundary conditions for the present problem are

$\mathrm{y}=0, \mathrm{~T}=\mathrm{T}_{\mathrm{m}}, \mathrm{k} \frac{\partial \mathrm{T}}{\partial \mathrm{y}}=\rho\left[\mathrm{h}_{\mathrm{st}}+\mathrm{C}_{\mathrm{s}}\left(\mathrm{T}_{\mathrm{m}}-\mathrm{T}_{0}\right)\right] \mathrm{v}$

and $\mathrm{y} \rightarrow \infty, \mathrm{T} \rightarrow \mathrm{T}_{\infty}, \mathrm{u}=\mathrm{u}_{\infty}$

Where $\mathrm{h}_{\mathrm{sf}}$ and $\mathrm{C}_{\mathrm{s}}$ are latent heat of the solid and specific heat of the solid phases respectively and $\mathrm{u}_{\infty}$ is the assisting external flow velocity, $\mathrm{k}=\alpha \rho \mathrm{C}_{\mathrm{p}}$ is the effective thermal conductivity of the porous medium. The boundary condition Eq. 5 means that the temperature on the plate is constant and thermal flux of heat conduction to the melting surface is equal to the sum of the heat of melting and the heat required for raising the temperature of solid to its melting temperature $T_{m}$.

Introducing the stream function $\psi$ with $\mathrm{u}=\frac{\partial \psi}{\partial y}$, and $\mathrm{v}=-\frac{\partial \psi}{\partial \mathrm{x}}$.

The continuity Eq. 1 will be satisfied and the Eq. 2 and Eq. 3 transform to

$$
\begin{aligned}
& \frac{\mu}{K} \frac{\partial^{2} \psi}{\partial y^{2}}+2 \frac{\mu}{K} \frac{\mathrm{C} V}{v} \frac{\partial \psi}{\partial y} \frac{\partial^{2} \psi}{\partial y^{2}}+\sigma B_{0}^{2} \frac{\partial^{2} \psi}{\partial y^{2}}=-\rho_{\infty} g \beta \frac{\partial T}{\partial y} \\
& \frac{\partial y}{\partial y} \frac{\partial T}{\partial x}-\frac{\partial y}{\partial x} \frac{\partial T}{\partial y}=\frac{\partial}{\partial y}\left[\left(\alpha_{m}+\gamma \frac{\partial y}{\partial y} d\right) \frac{\partial T}{\partial y}\right]+ \\
& \frac{4 \sigma_{K}}{a p C_{D^{a}}} \frac{\partial}{\partial y}\left(\frac{\partial T^{4}}{\partial y}\right)+\frac{a Q_{0}}{k} \\
& \text { (T- } \mathrm{T}_{\mathrm{m}} \text { ) }
\end{aligned}
$$

Introducing the similarity variables as

$$
\Psi=\mathrm{f}(\eta)\left(\alpha_{\mathrm{m}} \mathrm{u}_{\infty} \mathrm{x}\right)^{1 / 2}, \quad \eta=\left(\frac{\mathrm{u}_{\infty x}}{a_{\mathrm{m}}}\right)^{1 / 2}\left(\frac{\mathrm{y}}{\mathrm{x}}\right), \quad \theta(\eta)=
$$

$\frac{T-T_{m}}{T_{m}-T_{m}}$, the momentum equation Eq. 7 and energy equation Eq. 8 are reduced to

$$
\begin{aligned}
& \left(1+\mathrm{MH}+\mathrm{Ff}^{1}\right) \mathrm{f}^{11}+\frac{\mathrm{Ra}_{\mathrm{x}}}{\mathrm{Pe}_{\mathrm{x}}} \theta^{1}=0 \\
& \left(1+\mathrm{Df}^{1}\right) \theta^{11}+\left(\frac{1}{2} \mathrm{f}+\mathrm{Df}^{11}\right) \theta^{1}+\frac{4}{a} \\
& \mathrm{R}\left[\left(\theta+\mathrm{C}_{\mathrm{r}}\right)^{\mathrm{a}} \theta^{11}+3 \theta^{1^{2}}\left(\theta+\mathrm{C}_{\mathrm{r}}\right)^{2}\right]+(1+\mathrm{D}) \mathrm{r} \theta=0
\end{aligned}
$$

(10)

Where the prime symbol denotes the differentiation with respect to the similarity variable $\eta$ and $\mathrm{Ra}_{\mathrm{x}} / \mathrm{Pe}_{\mathrm{x}} \mathrm{is}$ the mixed convection parameter, $\mathrm{Ra}_{\mathrm{x}}=$

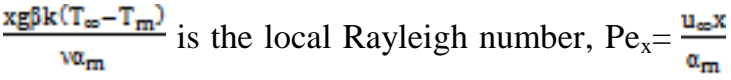
is the local Peclet number, $F=\frac{2 \mathrm{C}_{\mathrm{NKu}}}{\mathrm{v}}$ is the nonDarcian parameter, $\mathrm{D}=\frac{\mathrm{Pdu_{m }}}{\mathrm{a}_{\mathrm{m}}}$ is the dispersion parameter, $C_{r}=\frac{T_{m}}{T_{\infty}-T_{m}}$ is the temperature ratio, $M H$ $=\frac{G B_{0}^{2} K}{p v}$ is magnetic parameter, $R=\frac{4 \sigma_{R}\left(T_{s w}-T_{m}\right)^{3}}{k g}$ the radiation parameter, and $\mathrm{r}=\frac{\mathrm{Q}_{0} \mathrm{x}^{2}}{\mathrm{kPe_{ \textrm {x } }}}$ is the dimensionless heat generation or absorption parameter $(r<0$ corresponds to heat absorption and $r>0$ corresponds to heat generation).

Taking into consideration, the thermal dispersion effect together with melting, the boundary conditions Eq. 5 and Eq. 6 take the form $\eta=0, \theta=0, \mathrm{f}(0)+\left\{1+\mathrm{Df}^{1}(0)\right\} 2 \mathrm{M} \theta^{1}(0)=0$. and $\eta \rightarrow \infty, \theta=1, \mathrm{f}^{1}=1$. where $\mathrm{M}=\frac{\mathrm{c}_{\mathrm{f}}\left(\mathrm{T}_{\mathrm{w}}-\mathrm{T}_{\mathrm{m}}\right)}{\mathrm{h}_{\mathrm{sf}}+\mathrm{c}_{\mathrm{s}}\left(\mathrm{T}_{\mathrm{m}}-\mathrm{T}_{0}\right)}$ is the melting parameter. The local heat transfer rate from the surface of the plane is given by $q_{w}=-k\left(\frac{\partial T}{\partial y}\right)_{y=0}$

The Nusselt number is $N_{u}=\frac{h x}{k}=\frac{q_{w} x}{k\left(T_{m}-T_{m b}\right)}$, Where $h$ is the local heat transfer coefficient and $\mathrm{k}$ is the effective thermal conductivity of the porous medium, which is the sum of the molecular thermal conductivity $\mathrm{k}_{\mathrm{m}}$ and the dispersion thermal conductivity $\mathrm{k}_{\mathrm{d}}$.

The modified Nusselt number is obtained as $\frac{\mathbb{N}_{\mathbb{L}_{\mathrm{X}}}}{\left(\mathrm{Pe}_{\mathrm{X}}\right)^{1 / 2}}=\left[1+\frac{4}{\mathrm{~g}} \mathrm{R}\left(\theta(0)+\mathrm{C}_{\mathrm{r}}\right)^{\mathrm{a}}+\mathrm{D} \mathrm{f}^{1}(0)\right] \theta^{1}(0)$

\section{SOLUTION PROCEDURE}

The dimensionless equations Eq. 9 and Eq. 10 together with the boundary conditions Eq.11 and Eq.12 are solved numerically by means of the fourth order Runge-Kutta method coupled with double shooting technique. The solution thus obtained is matched with the given values of $\mathrm{f}^{1}(\infty)$ and $\theta(0)$. In addition, the boundary condition $\eta \rightarrow \infty$ is approximatedby $\eta_{\max }=8$ which is found sufficiently large for the velocity and temperature to approach the relevant free stream properties.Numerical computations are carried out for $\mathrm{F}=1 ; \mathrm{D}=0,0.5,1 ; \mathrm{Ra} / \mathrm{Pe}=1,-1 ; \mathrm{M}=0,0.8,2$; $\mathrm{R}=0.5 ; \mathrm{Cr}=0.1,0.5,1 ; \mathrm{r}=-0.1,0,0.1$. 


\section{RESULTS AND DISCUSSION}
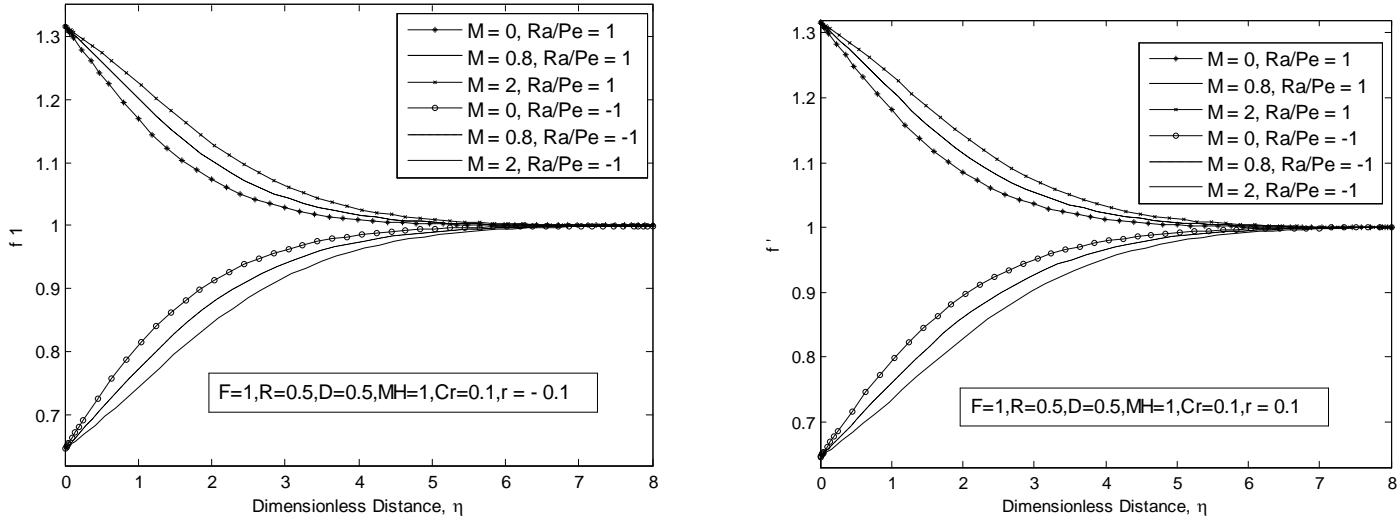

Fig. 2. Velocity profiles for different values of melting parameter with heat absorption $(r=-0.1)$ and generation $(\mathrm{r}=0.1)$.

The velocity profiles for aiding and opposing flows are shown in Fig. 2 for different melting parameter values in the presence of heat absorption and heat generation coefficient ' $r$ '. In aiding flow the increase in melting parameter leads to increase in velocity of the fluid both in the presence of heat absorption and heat generation coefficient. This effect is found opposite for the opposing flow.
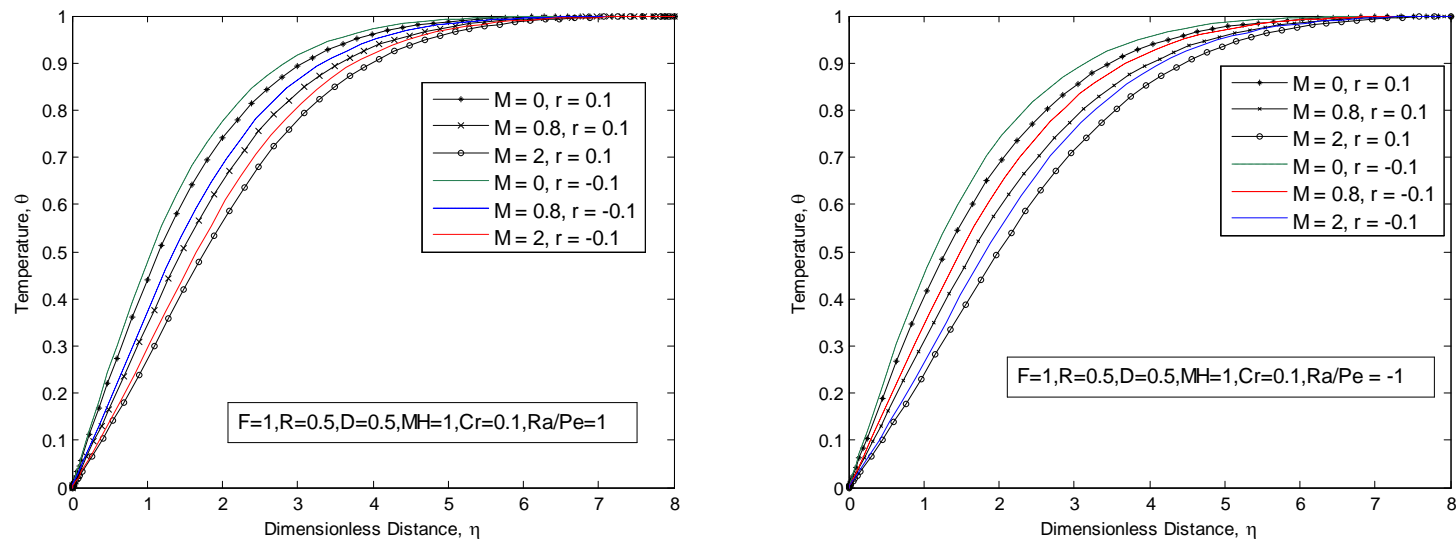

Fig. 3 Temperature profiles for different values of melting parameter in the presence of heat absorption $(r=-0.1)$ and heat generation $(r=0.1)$ in aiding and opposing flows.

Figure 3 shows the effect of melting parameter on temperature profiles in the presence of heat absorption and heat generation coefficient ' $r$ ' in aiding and opposing flows. In both flow cases, the same effect is found. The temperature decreases with the increase in the melting parameter both in the presence of heat absorption and heat generation coefficient. It is also noted that as the ' $r$ ' value increases, the temperature of the fluid decreases at a fixed melting parameter value.The velocity profiles for aiding and opposing flows are shown in Fig. 4 for different thermal dispersion parameter values in the presence of heat absorption and heat generation coefficient ' $r$ '. In aiding flow the increase in thermal dispersion parameter leads to increase in velocity of the fluid both in the presence of heat absorption and heat generation coefficient. This effect is found opposite for the opposing flow.

Figure 5 shows the effect of thermal dispersion parameter on temperature profiles in the presence of heat absorption and heat generation coefficient ' $r$ ' in aiding and opposing flows. In both flow cases, the same effect is found. The temperature decreases with the increase in the melting parameter both in the presence of heat 
absorption and heat generation coefficient. It is also noted that as the ' $r$ ' value increases, the

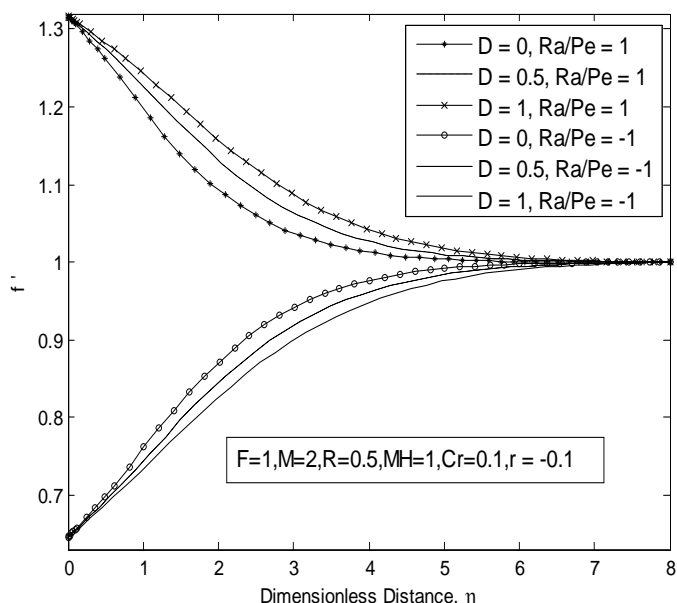

temperature of the fluid decreases at a fixed melting parameter value.

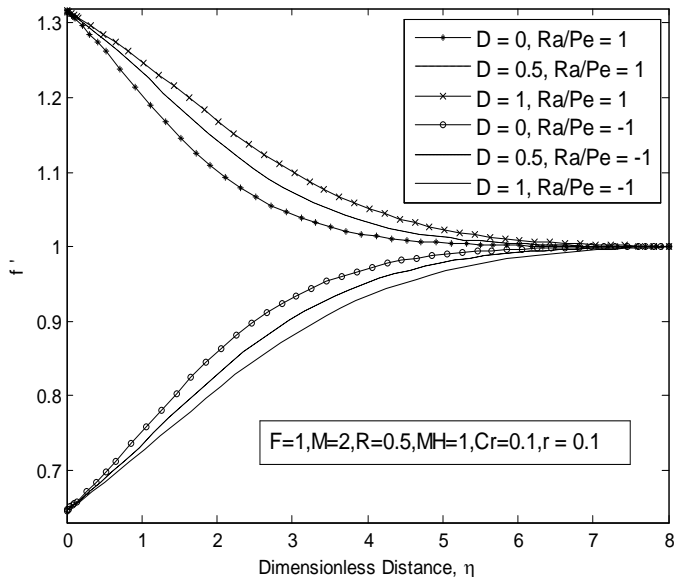

Fig. 4 Velocity profiles for different values of thermal dispersion parameter with heat absorption $(r=-0.1)$ and generation $(r=0.1)$.
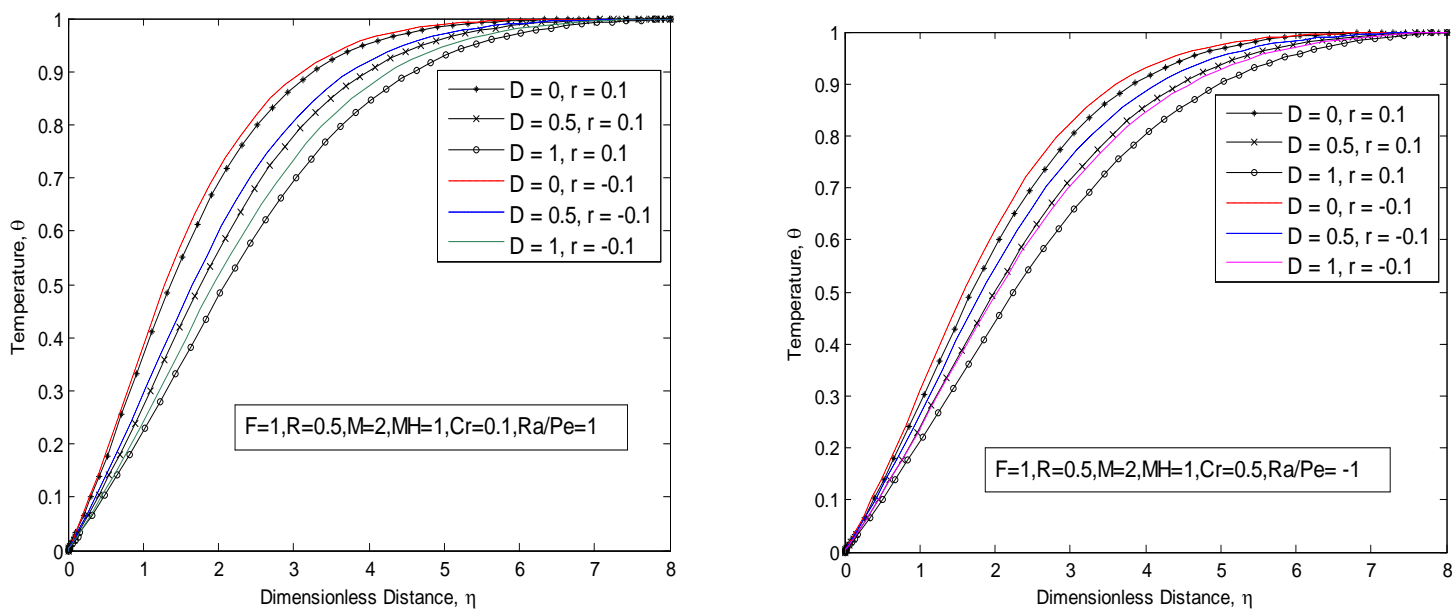

Fig. 5 Temperature profiles for different values of thermal dispersion parameter in the presence of heat absorption $(r=-0.1)$ and heat generation $(r=0.1)$ in aiding and opposing flows.

Figure 6 depict the effects of the heat generation or absorption coefficient ' $r$ ' on the velocity profiles in aiding and opposing flows without and with thermal dispersion, in the case of melting parameter $\mathrm{M}=2$, radiation parameter $\mathrm{R}=0.5$, flow inertia parameter $\mathrm{F}=1$. The presence of a heat generation source and heat absorption sink in the flow are represented by positive value $r=0.1$ and negative value $r=-0.1$ respectively. In aiding flow, It is noted that the velocity profile increases with the increase of ' $r$ ' value in both the cases of absence and presence of thermal dispersion effect. But the effect is found opposite in opposing flow in the presence and absence of thermal dispersion.

Figure 7 illustrate the influence of the heat generation or absorption coefficient ' $r$ ' on the temperature profiles in aiding and opposing flows without and with thermal dispersion, in the case of melting parameter $\mathrm{M}=2$, radiation parameter $\mathrm{R}=0.5$, flow inertia parameter $F=1$. It is noted that the temperature profile decreases with the increase of ' $r$ ' value in the absence as well as the presence of thermal dispersion effect both in aiding and opposing flow cases. The velocity profiles for aiding and opposing flows are shown in Fig. 8 for different temperature ratio parameter values in the presence of heat absorption and heat generation coefficient ' $r$ '. In aiding flow the increase in temperature ratio parameter leads to increase in velocity of the fluid both in the presence of heat absorption and heat generation coefficient. This effect is found opposite for the opposing flow. 

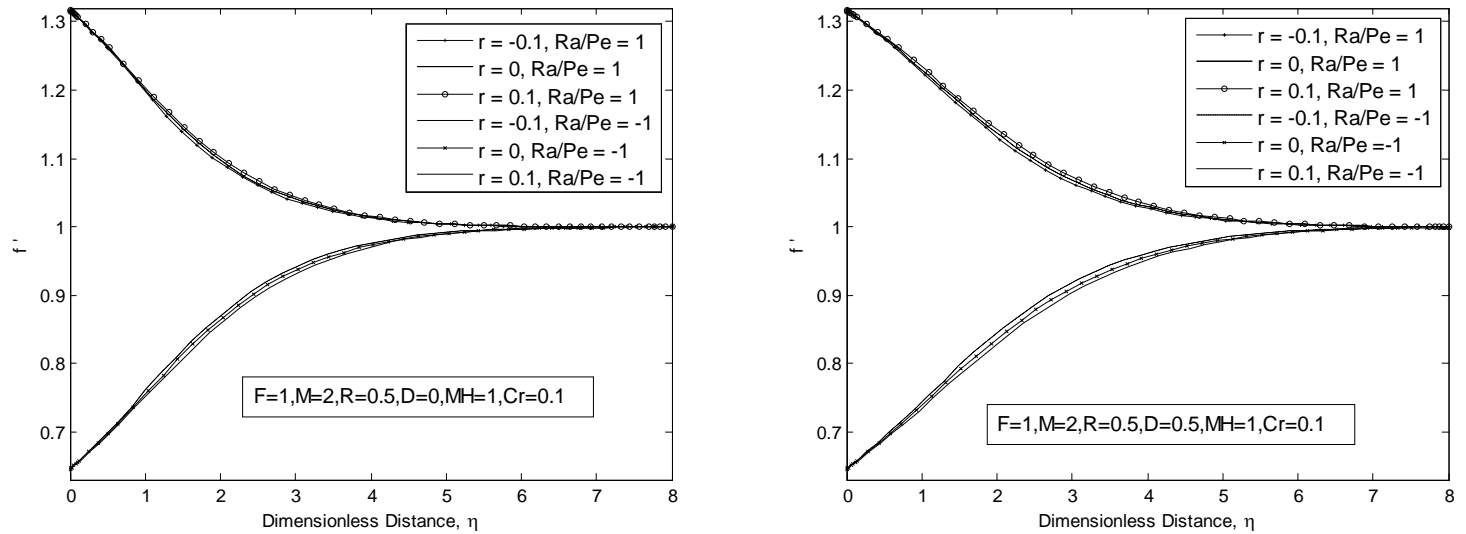

Fig. 6 Velocity profiles for different values of ' $r$ ' without and with dispersion in aiding and opposing flows
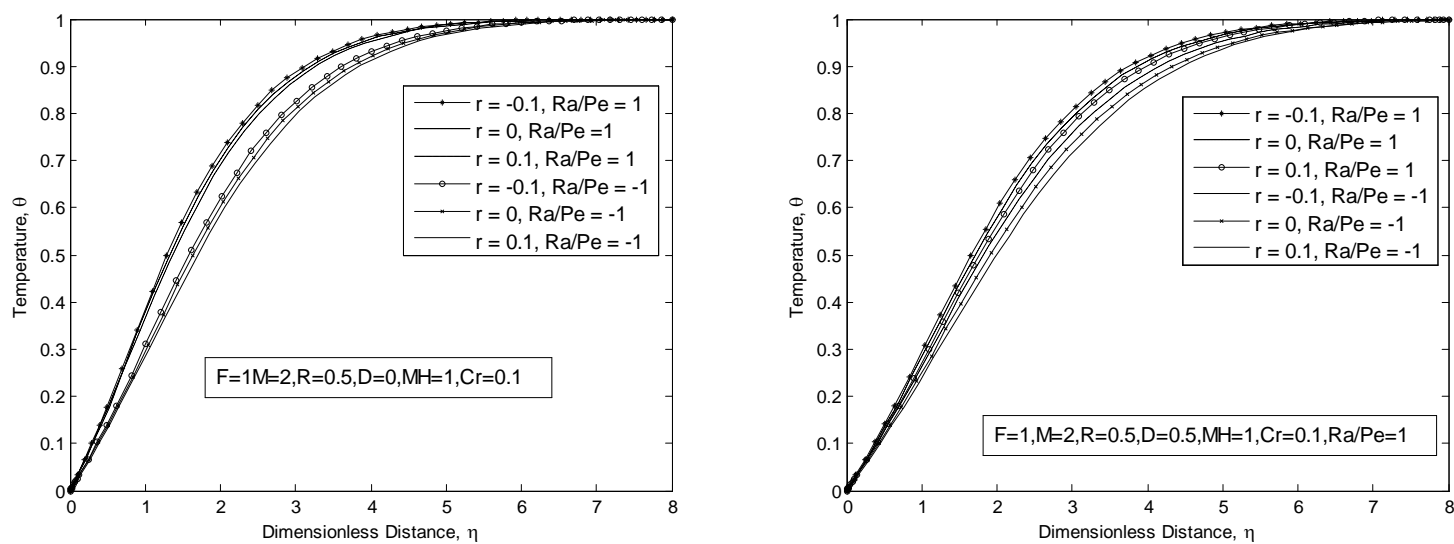

Fig. 7 Temperature profiles for different values of ' $r$ ' in the absence and presence of thermal dispersion in aiding and opposing flows.
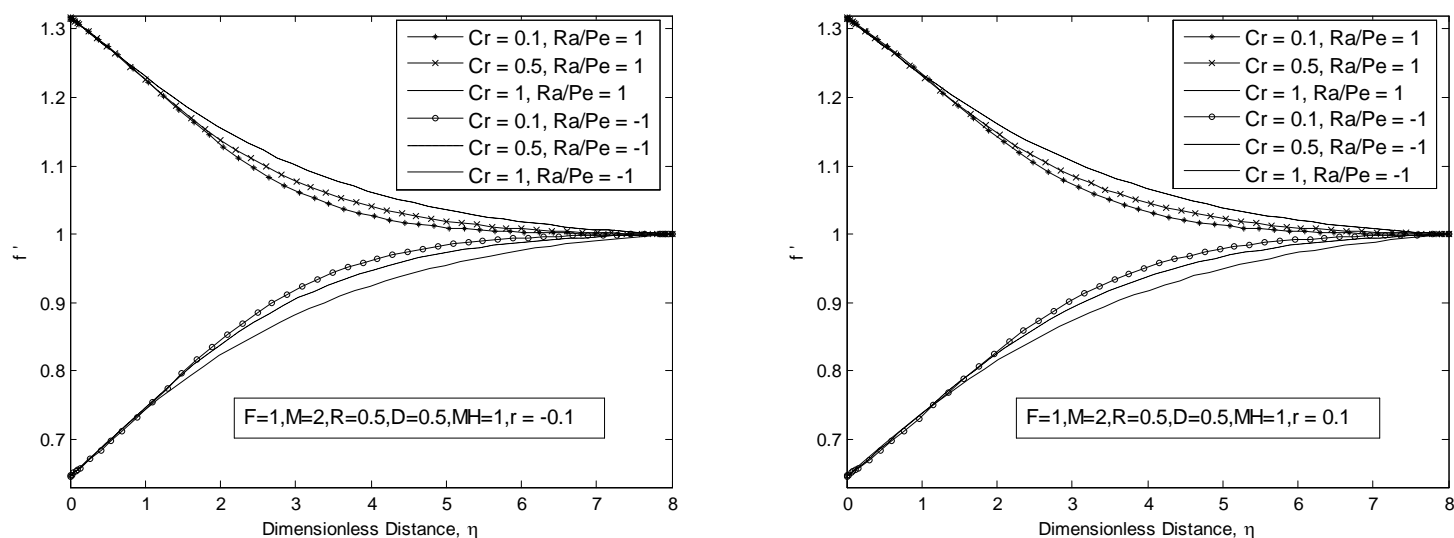

Fig. 8 Velocity profiles for different values of temperature ratio parameter with heat absorption $(r=-0.1)$ and generation $(\mathrm{r}=0.1)$.

Figure 9 show the effect of temperature ratio parameter on temperature profiles in the presence of heat absorption and heat generation coefficient ' $r$ ' in aiding and opposing flows. In both flow cases, the same effect is found. The temperature decreases with the increase in the temperature ratio parameter both in the presence of heat absorption and heat generation coefficient. It is also noted that as the ' $r$ ' value increases, the temperature of the fluid decreases at a fixed temperature ratio parameter value. 

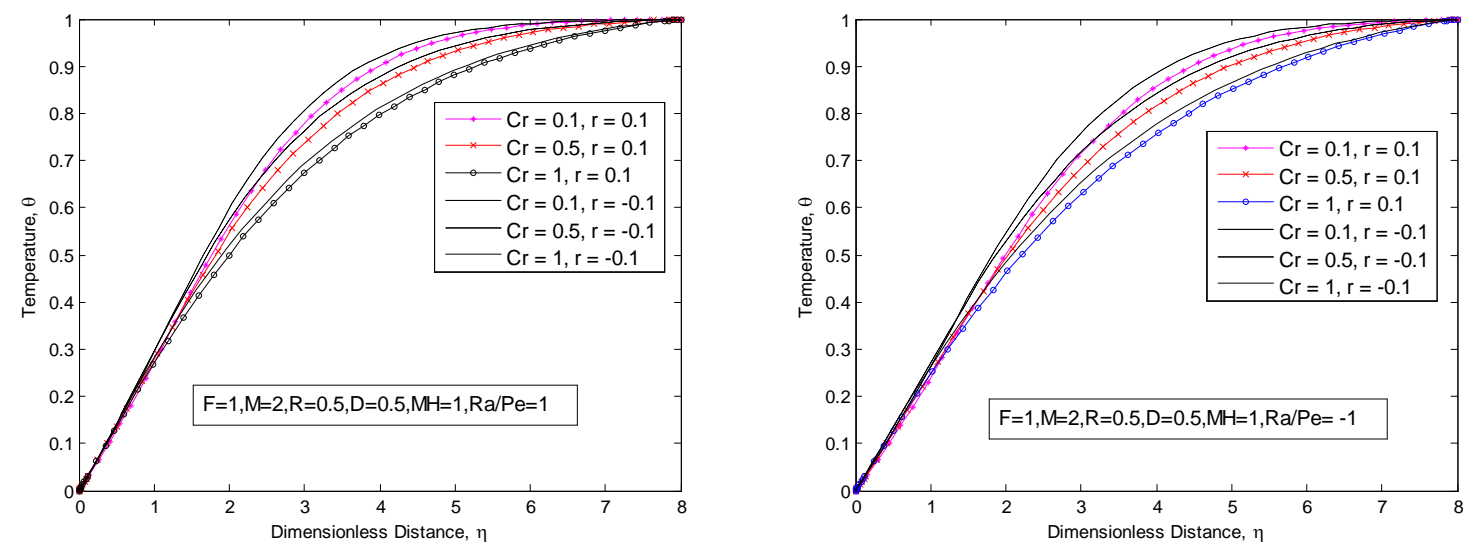

Fig. 9 Temperature profiles for different values of temperature ratio parameter in the presence of heat absorption $(r=-0.1)$ and heat generation $(r=0.1)$ in aiding and opposing flows.

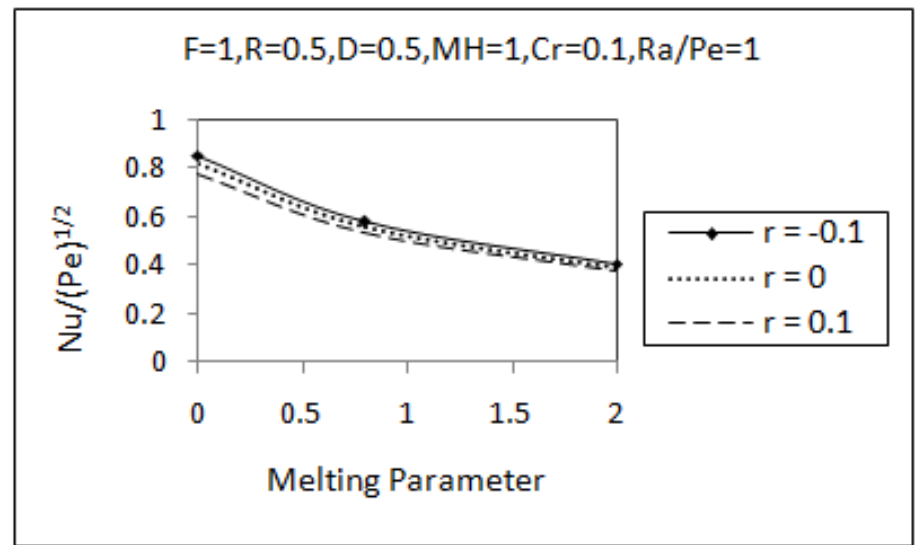

Fig. 10. Variation of local Nusselt number with the melting parameter for different values of heat absorption or generation coefficient in aiding flow.

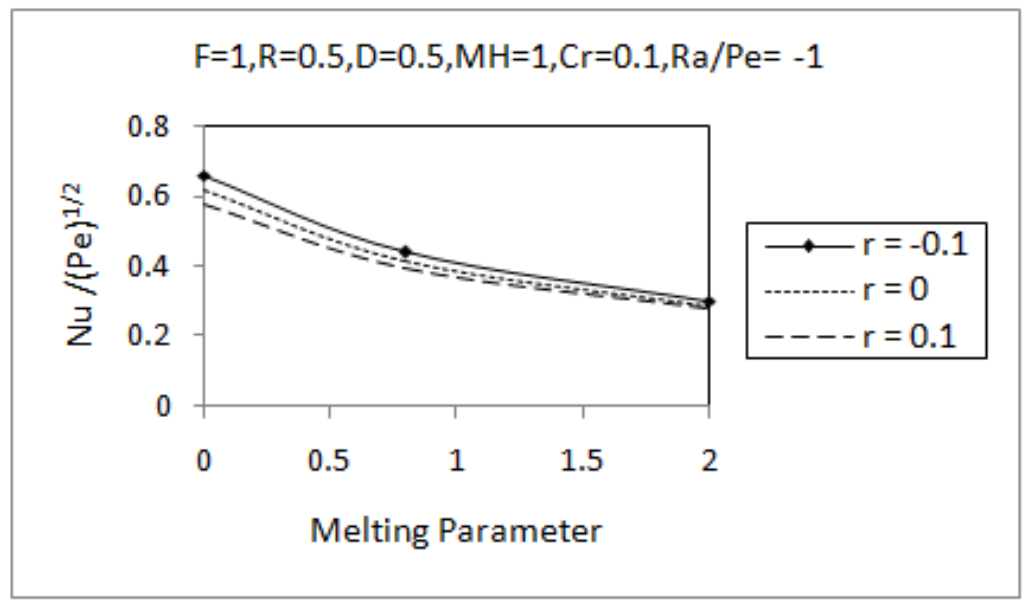

Fig. 11 Variation of local Nusselt number with the melting parameter for different values of heat absorption or generation coefficient in opposing flow.

Figures 10 and 11 shows the effect of heat absorption or generation parameter on the Nusselt number given in Eq. (13) for different values of melting parameter for aiding and opposing flows respectively. The same effect is found in both flow cases. The increase in the value of absorption or generation parameter causes the decrease in Nusselt number, whereas increase in the melting parameter results in the decrease in Nusselt number. 
Figures12 and 13 shows the effect of temperature ratio parameter in the presence of heat absorption on the Nusselt number for different values of melting parameter for aiding and opposing flows respectively. The increase in the value of temperature ratio parameter causes the increase in Nusselt number, whereas increase in the melting parameter results in the decrease in Nusselt number in both flow cases. Figures 14 and 15 shows the effect of temperature ratio parameter in the presence of heat generation on the Nusselt number for different values of melting parameter for aiding and opposing flows respectively. The increase in the value of temperature ratio parameter causes the increase in Nusselt number, whereas increase in the melting parameter results in the decrease in Nusselt number in both flow cases. Figures 16 and 17 shows the effect of thermal dispersion parameter in the presence of heat absorption on the Nusselt number for different values of melting parameter for aiding and opposing flows respectively. The increase in the value of thermal dispersion parameter causes the increase in Nusselt number, whereas increase in the melting parameter results in the decrease in Nusselt number in both flow cases. Figures 18 and 19 shows the effect of thermal dispersion parameter in the presence of heat generation on the Nusselt number for different values of melting parameter for aiding and opposing flows respectively. The increase in the value of thermal dispersionparameter causes the increase in Nusselt number, whereas increase in the melting parameter results in the decrease in Nusselt number in both flow cases.

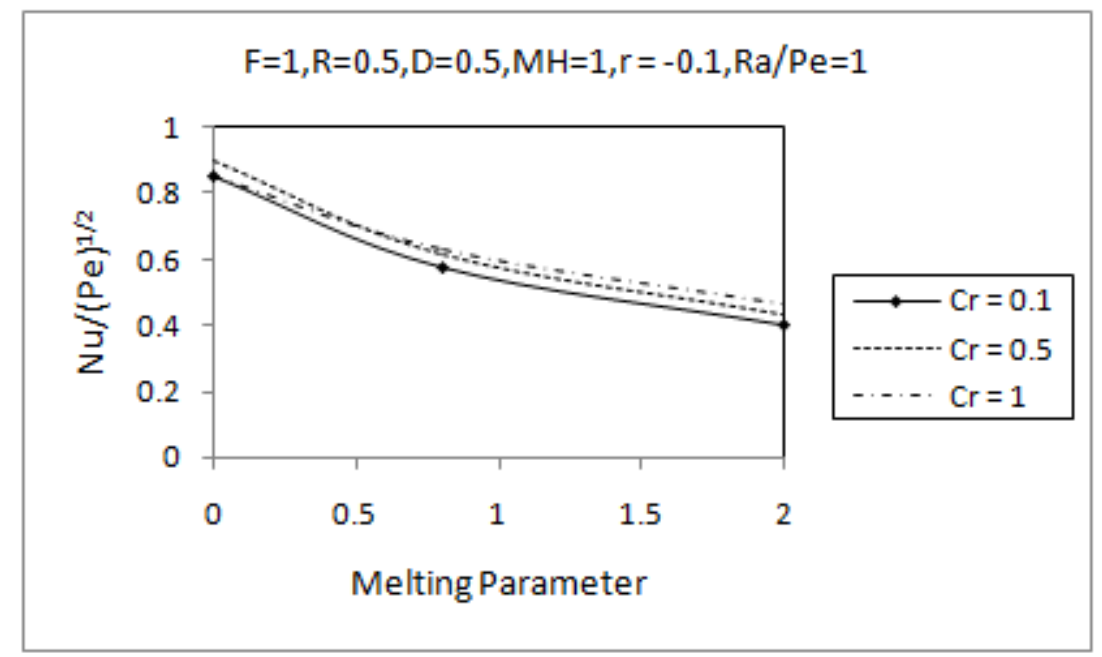

Fig. 12. Variation of local Nusselt number with the melting parameter for different values of temperature ratio parameter in the presence of heat absorptionin aiding flow.

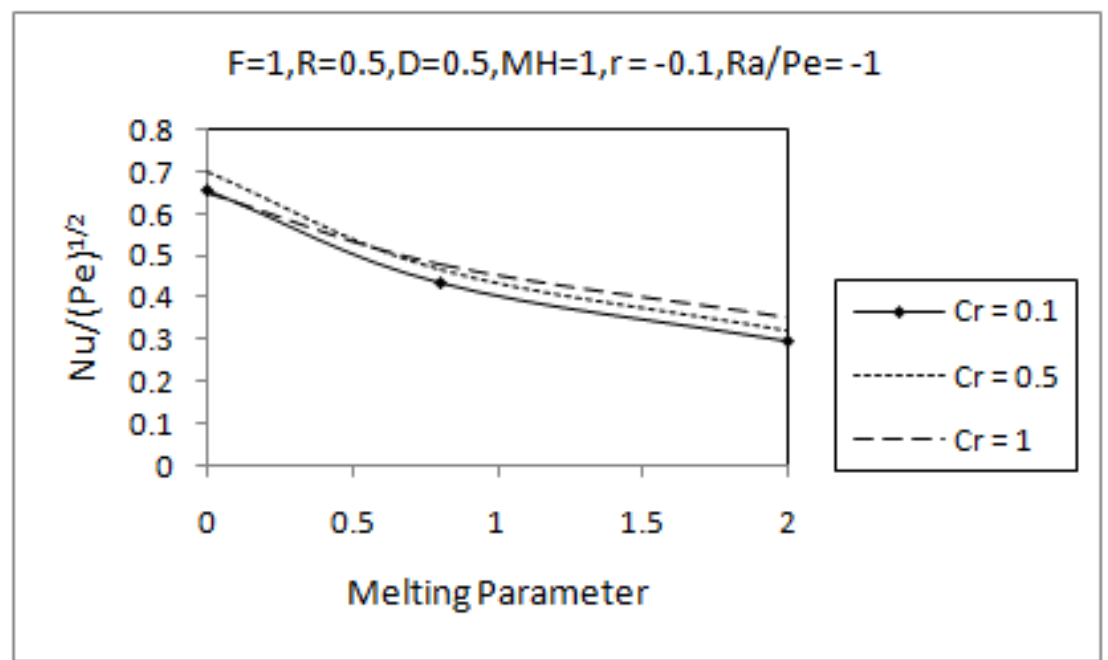

Fig. 13. Variation of local Nusselt number with the melting parameter for different values of temperature ratio parameter in the presence of heat absorption in opposing flow. 


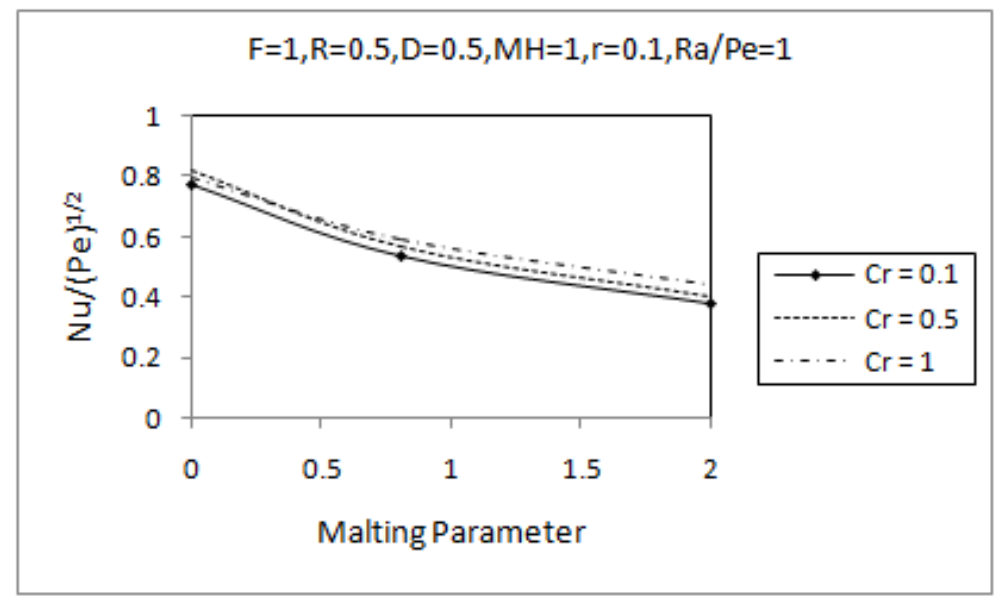

Fig. 14. Variation of local Nusselt number with the melting parameter for different values of temperature ratio parameter in the presence of heat generation in aiding flow.

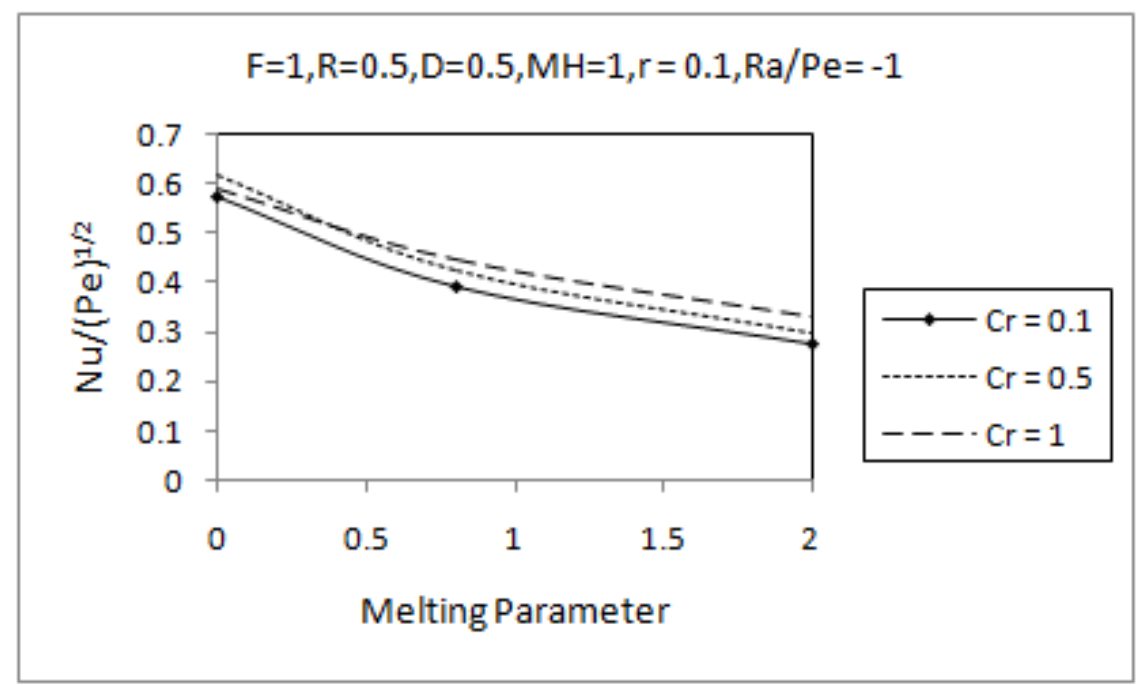

Fig. 15. Variation of local Nusselt number with the melting parameter for different values of temperature ratio parameter in the presence of heat generation in opposing flow.

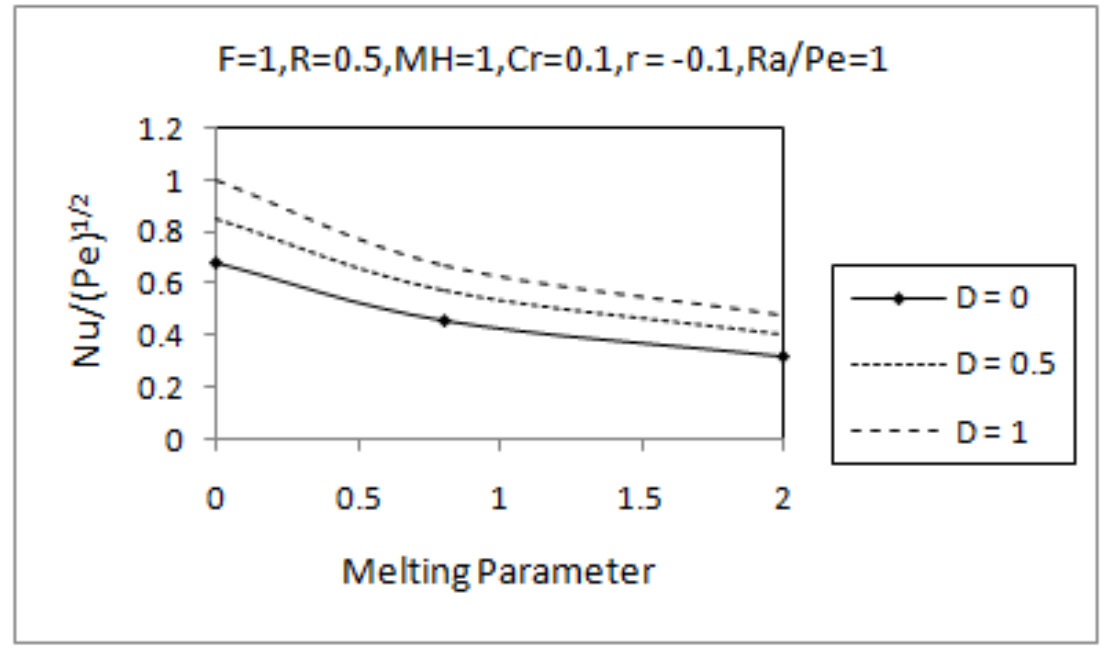

Fig. 16. Variation of local Nusselt number with the melting parameter for different values of thermal dispersion parameter in the presence of heat absorptionin aiding flow. 


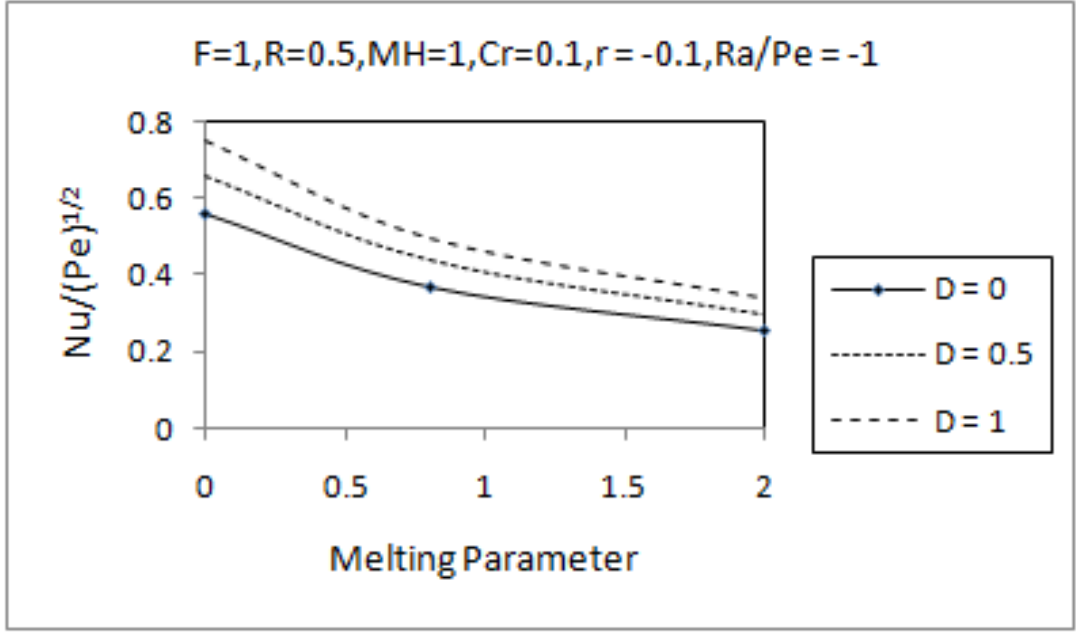

Fig. 17.Variation of local Nusselt number with the melting parameter for different values of thermal dispersion parameter in the presence of heat absorptionin opposing flow.

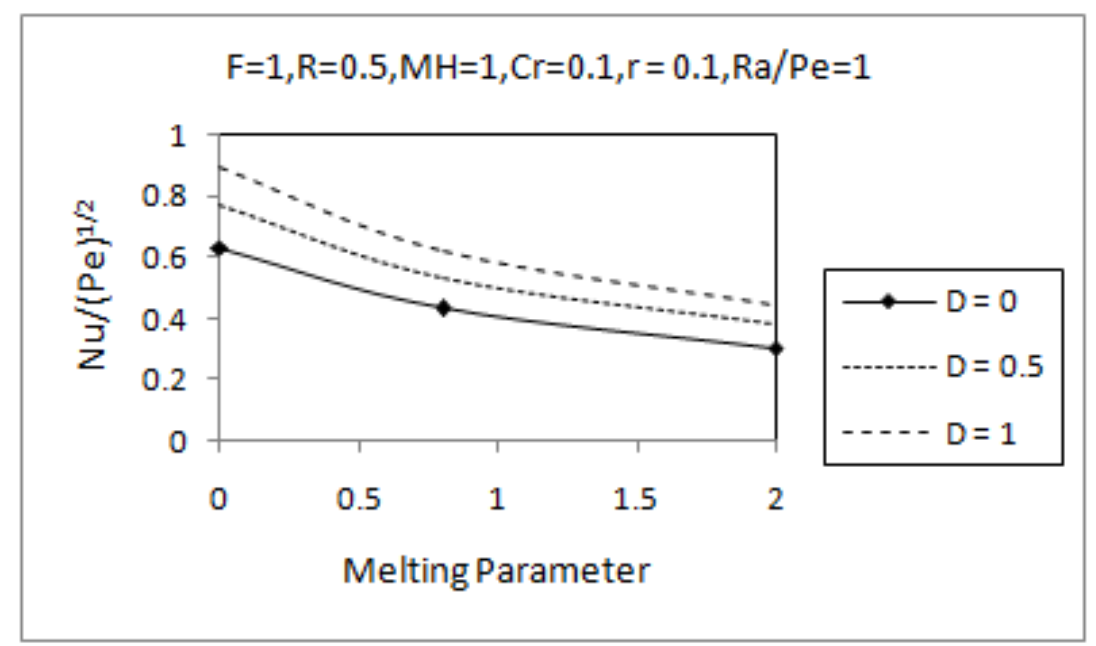

Fig. 18. Variation of local Nusselt number with the melting parameter for different values of thermal dispersion parameter in the presence of heat generation in aiding flow.

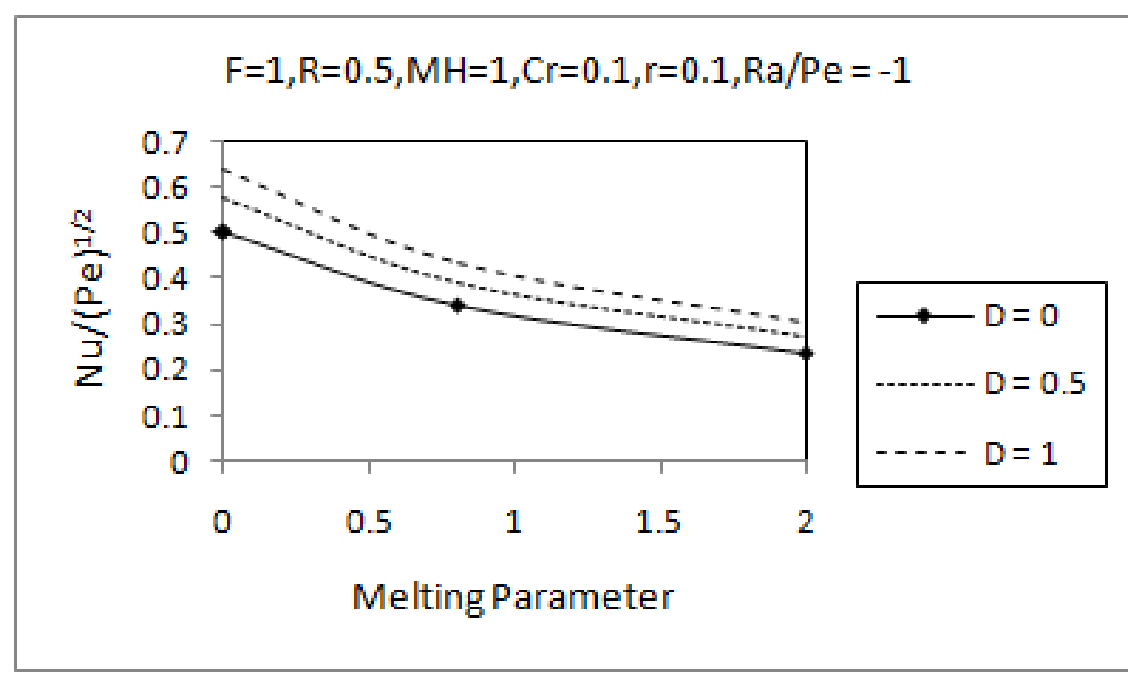

Fig. 19.Variation of local Nusselt number with the melting parameter for different values of thermal dispersion parameter in the presence of heat generation in opposing flow. 


\section{CONCLUSION}

The melting phenomenon has been analyzed in the presence of heat absorption and heat generation coefficient with mixed convection flow and heat transfer in a saturated non-Darcy porous medium considering the effects of thermal dispersion, thermal radiation and applied magnetic field by taking Forcheimer extension in the flow equations. Numerical results for the velocity and temperature profiles as well as the heat transfer rate as a function of Nusselt number are obtained for aiding and opposing flows and the same are presented graphically. Same results are obtained in the presence of heat absorption and heat generation coefficient. This study shows that the increase in melting, thermal dispersion, heat absorption or generation and temperature ratio parameters tend to increase / decrease the velocity within the boundary in aiding / opposing flow. Further, it is noticed that the temperature decreases with the increase in melting, thermal dispersion, heat absorption or generation and temperature ratio parameters in both flow cases. Moreover it is found that the rate of heat transfer decreases with the increase in melting parameter while it increases with the increase in thermal dispersion and temperature ratio parameter values in both aiding and opposing flows. It is also noticed that the Nusselt number decreases with the increase in heat absorption or generation parameter value in both flow cases.

\section{REFERRENCES}

[1] Leonard Roberts, "On the Melting of a Semi - Infinite Body of Ice placed in a Hot Stream of Air, J.Fluid Mech.4, pp.505-528, 1958.

[2] MKazmierczak, D Poulikakos and I Pop, Melting from a flat plate embedded in a porous medium in the presence of steady natural convection, Numer Heat Transfer, 10, 1986, pp. 571-582.

[3] P Cheng, Combined Free and Forced Boundary Layer Flows about Inclined Surfaces in a Porous Medium, Int. J Heat Mass Transfer, 20, 1977, pp. 807-814.

[4] J H Merkin, Mixed Convection Boundary Layer Flow on a vertical surface in a Saturated Porous Medium, J. Engng. Math, 14, 1980, pp. 301-313.

[5] A J Chamkha, Non-Darcy Hydro Magnetic Free Convection from a Cone and a Wedge In Porous Media, Int. Comm. Heat Mass Transfer, 23, 1996, pp. 875887.

[6] PVSN Murthy, P Singh, Thermal dispersion effects on non-Darcy Convection over a Cone, Comp. Math.
With Applications, 40, 2000, pp. 14331444.

[7] Alinv. Rosca. Natalia c. Rosca. Ian pop, Mixed convection heat and mass transfer from a vertical surface embedded in a saturated porous medium, Transp porous med(2015) 109:279-295

[8] J.H.Merkin, I.Pop, S.Ahmad, Note on melting effect on flat mixed convection boundary layer over avertical surface embedded in a porous medium, International journal of heat and mass transfer 84,(2015) 786-790

[9] Syakila Ahmad, Ian pop, Melting effect on mixed convection boundary layer flow about a vertical surface embedded in a porous medium: opposing flows case, Transp porous med(2014) 102:317-323.

[10] IA Hassanien, TH Al-arabi, Non-Darcy Unsteady Mixed Convection Flow Near the Stagnation Point on a Heated Vertical Surface Embedded in a Porous Medium with Thermal Radiation and Variable Viscosity, Commun. Nonlinear Sci. Numer. Simulate, 14, 2009, pp. 13661376.

[11] PVSN Murthy, S Mukherjee, D.srinivasacharya and P.V.S.S.R.k, Warangal,Combined Radiation and Mixed Convection from a Vertical Wall with Suction/Injection in a Non-Darcy Porous Medium, Acta Mech., 168, 2004, pp. 145156.

[12] AJ Chamkha, AF Al-Mudhaf, I Pop, Effect of Heat Generation or Absorption on Thermophoretic Free Convection Boundary Layer from a Vertical Flat Plate Embedded in a Porous Medium, Int. Comm. Heat Mass Transfer, 33, 2006, pp. 1096-1102.

[13] BDCN Prasad, K Hemalatha, Non-Darcy mixed convection with thermal dispersionradiation in a saturated porous medium, The Open Transport Phenomina Journal, 2, 2010, pp. 109-115.

[14] Ali J Chamkha, Heat and Mass Transfer for a Non-Newtonian Fluid Flow Along a Surface Embedded in a Porous Medium with Uniform Wall Heat and Mass Fluxes and Heat Generation or Absorption, International Journal of energy, 3, vol. 1, 2007, pp. 97-104.

[15] Ali J Chamkha. al, Melting and Radiation Effects on Mixed Convection from a Vertical Surface Embedded in a NonNewtonian Fluid Saturated Non-Darcy Porous Medium for Aiding and Opposing External Flows, International Journal of 
the Physical Sciences, 5(7), 2010, pp. 1212-1224.

[16] O A Plumb, The Effect of Thermal Dispersion on Heat Transfer in Packed Bed Boundary Layers, Proceedings of $1^{\text {st }}$ ASME/JSME, Thermal Engineering Joint Conferrence, 2, 1983, pp. 17-21.

[17] E M Sparrow, R D Cess, Radiation heat transfer, Washington Hemisphere, 1978.

[18] A Raptis, Radiation and Free Convection Flow Through a Porous Medium, Int. Commn. Heat Mass Transfer, 25, 1998, pp. 289-295 